\title{
Tunable Enhancement of Second-Harmonic Generation in Dual Graphene Optical Gratings
}

\author{
$\underline{\text { Jian Wei You }}^{1}$ and Nicolae C. Panoiu ${ }^{1}$ \\ ${ }^{1}$ University College London, Department of Electronic and Electrical Engineering, WC1E7JE, London, UK \\ e-mail address: j.you@ucl.ac.uk
}

\begin{abstract}
Employing geometry dependent plasmon resonances of graphene gratings, we design a graphene bi-layer optical grating, which can achieve several orders of magnitude enhancement of the second-harmonic generation (SHG) intensity. More importantly, this dual grating can act as an ultrafast optical switch as the SHG intensity can be readily controlled via gate voltage tuning.
\end{abstract}

\section{INTRODUCTION}

Because of large local optical field enhancements achievable in graphene via excitation of surface plasmons, this two-dimensional (2D) physical system [1] provides a promising material platform for enhancing nonlinear optical interactions at the nanoscale [2]-[4]. In this paper, we demonstrate that by matching plasmon resonances in mutually interacting graphene gratings separated by a thin dielectric spacer, the second-harmonic generation (SHG) can be enhanced by orders of magnitude. Moreover, by tuning the Fermi level of one of the graphene gratings, one can readily control the SHG. To be more specific, employing the dependence of the resonance wavelength of localized plasmons of graphene ribbons on their width, we design a dual graphene grating in such a way that the resonance wavelength of the first-order plasmon mode in one of the graphene gratings is equal to half of that of the first-order localized plasmons in the other grating that is optically coupled to the first one. This finding is particularly relevant in the context of technological applications, as graphene plays an increasingly important role in optoelectronics, chiefly due to the remarkable tunability of its optical properties via chemical doping or electric gating. In addition, graphene structures support localized and propagating surface plasmons, whose excitation is accompanied by a significant enhancement of the optical near-field. This unique potential to control at deep-subwavelength the optical field has open up a broad array of technological applications, including optical modulation, sensing, and ultrafast active photonic devices [5],[6].

\section{LINEAR OPTICAL RESPONSE OF DUAL GRAPHENE GRATINGS}

The physical structure considered in this study consists of a graphene bi-layer optical grating made of graphene ribbons, depicted in Fig. 1a, where the two graphene gratings are placed at the top and bottom facets of a dielectric spacer. The space is made of glass with a constant relative permittivity, $\epsilon_{s}=2.25$.
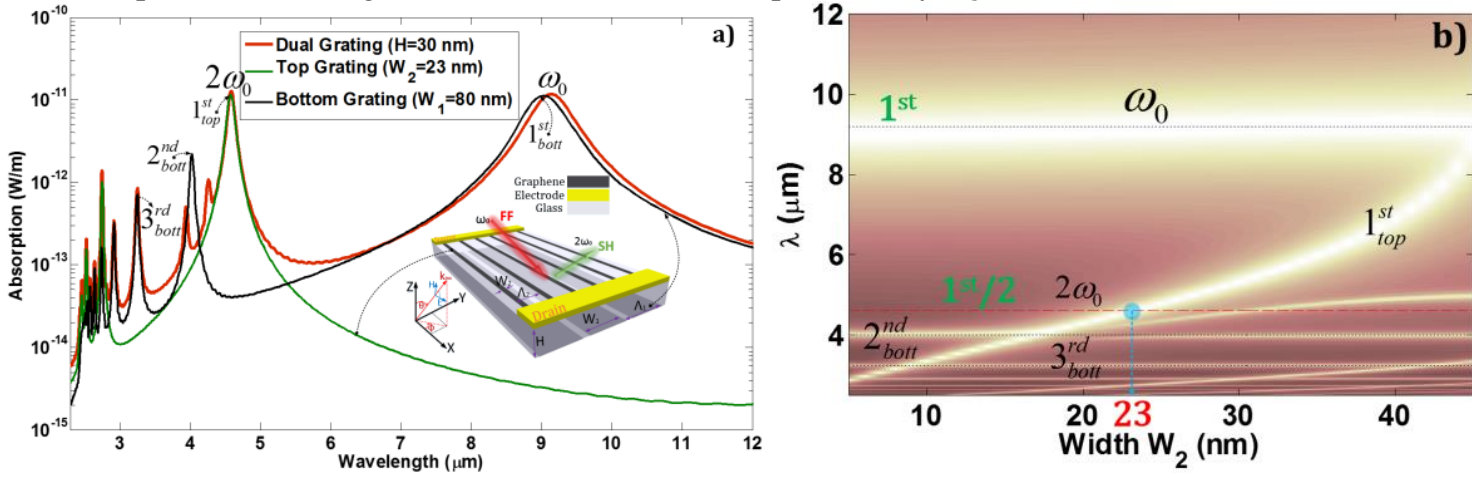

Fig. 1. Geometrical configuration and optimization of the proposed dual graphene optical gratings: (a) Enhancement of SHG in a dual graphene grating due to a double plasmon resonance at $\omega_{0}$ and $2 \omega_{0}$; (b) Optimization of the width $\mathrm{W}_{2}$ of the top graphene grating to achieve a double plasmon resonance as shown in Fig.1a. 
In our design, the two grating periods are, $\Lambda_{1}=100 \mathrm{~nm}$, and $\Lambda_{2}=50 \mathrm{~nm}$. The width of the bottom graphene grating $\mathrm{W}_{1}=80 \mathrm{~nm}$ and the thickness of the spacer is $H=30 \mathrm{~nm}$. In our simulations, the chemical potential of graphene is $0.6 \mathrm{eV}$, the relaxation time is $0.25 \mathrm{ps}$, and the temperature is $300 \mathrm{~K}$. The width of the top graphene grating $\left(\mathrm{W}_{2}\right)$ is the quantity we optimized to achieve a so-called double plasmon resonance [5] at the fundamental frequency (FF) $\omega_{0}$. To model the linear and nonlinear optical response of graphene gratings, we used an in-house developed numerical method implementing a combination of the generalized-source (GS) algorithm and finitedifferent time-domain method (FDTD) [7],[8].

The basic idea of SHG enhancement in the proposed dual graphene grating is illustrated in Fig. 1a. In this figure, the black line represents the linear absorption of the bottom graphene grating alone. This spectrum has a series of spectral peaks, which are due to the excitation of plasmon modes in the graphene ribbons. Among them, the firstorder plasmon resonance appears at FF $\omega_{0}$; however, there is no plasmon resonance at $2 \omega_{0}$. This leads to weak SHG in the combined optical grating, as the nonlinear optical field cannot be emitted efficiently. This issue can be overcome by using a so-called double plasmon resonance. More specifically, by varying the width $\left(\mathrm{W}_{2}\right)$ of the graphene ribbons, we design another graphene grating (the top grating) such that its first-order plasmon resonance appears at half of the wavelength of the first-order plasmon resonance of the bottom graphene grating. This property is illustrated by the green line in Fig. 1a.

To implement such a specially engineered double-resonance feature in a dual graphene grating, we scan the width $\left(\mathrm{W}_{2}\right)$ of the top graphene grating, and calculate the linear absorption spectra of the dual graphene grating. As illustrated in Fig. 1b, the bright band labeled with the symbol $1_{t o p}^{\text {st }}$ arises from the first-order plasmon resonance of the top graphene grating, and it has a cross point at $\mathrm{W}_{2}=23 \mathrm{~nm}$ with the dashed red line, which corresponds to half of the resonance wavelength of the first-order plasmon of the bottom graphene grating. Therefore, for this particular configuration of the combined optical grating $\left(\mathrm{W}_{1}=80 \mathrm{~nm}, \mathrm{~W}_{2}=23 \mathrm{~nm}\right.$, and $\left.H=30 \mathrm{~nm}\right)$, there is a double resonance at $\omega_{0}$, and $2 \omega_{0}$, as shown by the red line in Fig. $1 \mathrm{a}$.

\section{TUNABLE SWITCH AND NONLINEARITY ENHANCEMENT}

As is well known, the linear and nonlinear optical properties of graphene can be tuned via chemical doping or electrical gating. Based on this property of graphene, we develop a tunable switch to control the SHG enhancement in the proposed dual graphene optical grating.
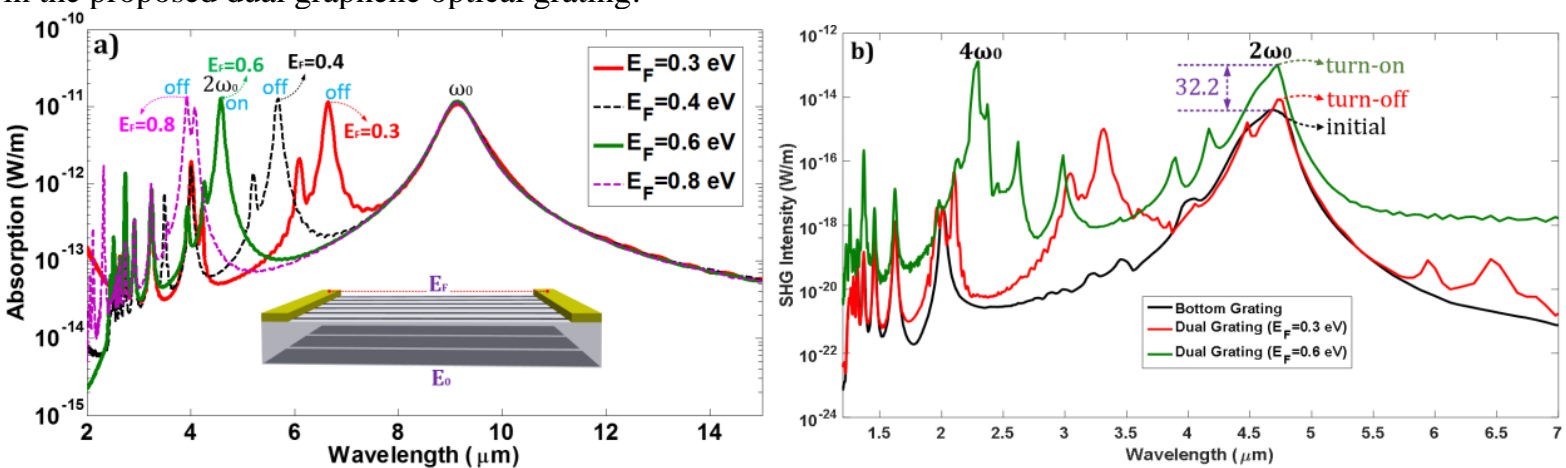

Fig. 2. Tunable switch and nonlinearity enhancement: (a) Tunability of the proposed dual graphene grating employed to a nonlinear optical switch; (b) Controllable SHG enhancement via changing the Fermi level of the top graphene grating.

As depicted in the inset in Fig. 2a, we place two electrodes on the top graphene grating to tune the Fermi level $\left(E_{F}\right)$ of the corresponding graphene ribbons, but leave unchanged the Fermi level $\left(E_{0}\right)$ of the bottom graphene grating at $0.6 \mathrm{eV}$. In this way, the first-order plasmon resonance of the bottom graphene grating will be at $\omega_{0}$, and we can tune the first-order plasmon resonance of the top graphene grating to bring it on and off resonance with the second-harmonic frequency $\left(2 \omega_{0}\right)$, by simply tuning the Fermi level $\mathrm{E}_{F}$. In other words, we can readily turn on or turn off the SHG intensity.

In order to illustrate these ideas, we present in Fig. 2b the SHG spectra calculated with our in-house developed GS-FDTD and repeated these calculations for several different values of the Fermi energy. The plots shown in 
Fig. $2 b$ reveal the switching property of this optical device. First, compared with the bottom grating, the SHG intensity in the dual graphene grating is enhanced by more than 30 times, if the first-order plasmon resonances in the two sub-gratings are tuned to $\omega_{0}$ and $2 \omega_{0}$ by changing the Fermi level of the top graphene grating to $0.6 \mathrm{eV}$. Then, we can turn off the SHG in the graphene grating, if the Fermi level of top graphene grating is modified to $0.3 \mathrm{eV}$. Therefore, this demonstrates that a tunable SHG intensity can be achieved in this optimized dual graphene grating by simply by varying the Fermi level of one of the graphene gratings (the top one in our illustration).

\section{CONCLUSION}

In conclusion, we have demonstrated that a tunable enhancement of SHG can be achieved in a dual graphene grating by properly designing the spectral locations of plasmon resonances of optically coupled graphene gratings, combined with gate voltage tuning. Because of the generality of the employed design principles, we expect that the ideas investigated here can also be applied to design other active graphene photonic nanodevices.

\section{ACKNOWLEDGEMENT}

This work was supported by the European Research Council, grant agreement no. ERC-2014-CoG-648328. The authors acknowledge the use of the UCL Legion High Performance Computing Facility (Legion@UCL) and associated support services in the completion of this work.

\section{REFERENCES}

[1] K. S. Novoselov, A. K. Geim, S. V. Morozov, D. Jiang, Y. Zhang, S. V. Dubonos, I. V. Grigorieva, and A. A. Firsov. "Electric field effect in atomically thin carbon films," Science, vol. 306, pp.666-669, Oct 2004.

[2] M. T. Manzoni, I. Silveiro, F. J. G. Abajo, and D. E. Chang. "Second-order quantum nonlinear optical processes in single graphene nanostructures and arrays," New J. Phys. vol. 17, p. 083031, May 2015.

[3] M. Weismann and N. C. Panoiu, "Theoretical and computational analysis of second- and third-harmonic generation in periodically patterned graphene and transition-metal dichalcogenide monolayers," Phys. Rev. B. vol. 94, p. 035435, 2016.

[4] J. W. You, J. You, M. Weismann, and N. C. Panoiu, "Double-resonant enhancement of third-harmonic generation in graphene nanostructures," Phil. Trans. R. Soc. A. vol. 375, p. 20160313, Feb 2017.

[5] F. Bonaccorso, Z. Sun, T. Hasan, and A. C. Ferrari. "Graphene photonics and optoelectronics," Nat. Photonics, vol. 4, pp.611-622, August 2010.

[6] F. Xia, T. Mueller, Y. M. Lin, A. Valdes-Garcia, and P. Avouris. "Ultrafast graphene photodetector," Nat. Nanotechnol., vol. 4, pp.839-843, Oct 2009.

[7] A. Taflove, Computational Electrodynamics: The Finite-Difference Time-Domain Method, 2nd ed., Norwood, MA: Artech House, 1995.

[8] J. W. You, S. R. Tan and T, J, Cui. "Novel Adaptive Steady-State Criteria for Finite-Difference Time-Domain Method," IEEE Trans. Microw. Theory Tech., vol. 62, no.12, Dec 2014. 